\title{
FORMULATION AND IN VITRO CHARACTERISATION OF SOYBEAN OIL-HPMCK4M BASED BIGEL MATRIX FOR TOPICAL DRUG DELIVERY
}

\author{
SHUBHAM MUKHERJEE*, SUTAPA BISWAS MAJEE, GOPA ROY BISWAS
}

Department of Pharmaceutical Technology, NSHM Knowledge Campus, Kolkata Group of Institutions, 124 B. L Saha Road, Kolkata 700053 Email: rx.shubhamm@gmail.com

Received: 08 May 2019, Revised and Accepted: 06 Jun 2019

\begin{abstract}
Objective: Hydrogels with scope for utilization in numerous fields possess limited applications due to problems in incorporating wide range of drugs and crossing the lipophilic barrier of the skin. Attempts to overcome these problems by developing organogel hold drawbacks. Challenges posed by drug lipophilicity or skin permeation can be solved by developing bigel formed via combination of lipophilic and hydrophilic gel phases in a definite proportion. The objective of the present study is to formulate and characterize matrix type bigel of soybean oil and HPMCK4M for topical drug delivery.

Methods: Four batches of bigels were developed with two organogel formulations of soybean oil containing 20 and $22 \%$ w/v Span 60 . Both organogels and bigels were examined for compatibility by FTIR spectroscopy, hemocompatibility and characterized for physical appearance, pH, rheological behavior and in vitro drug release pattern.

Results: FTIR study confirmed compatibility between paracetamol and components of organogel or bigel. The oily feel of organogels disappeared with bigels which possessed a creamy and smooth texture. Pseudoplastic behaviour was confirmed by Ostwald-de wale power-law model in both organogels and bigels. Improved drug release was observed in bigel (BG1) formulation containing 3\%w/v HPMCK4M and soybean oil based organogel with $20 \%$ w/v Span 60 as compared to the corresponding organogel (OG1). Organogels were foundto follow either zero-order kinetics (OG1) or KorsmeyerPeppasmodel (OG2) while the formation of matrix was exhibited in bigels with drug diffusion predominantly of non-Fickian type.
\end{abstract}

Conclusion: Therefore, bigels of soybean oil based organogel with HPMCK4M hydrogel formed gel matrix demonstrating improved drug release for topical application compared to organogel.

Keywords: Bigel, Higuchi model, HPMCK4M, non-Fickian diffusion, Organogel, pseudoplastic flow, Soybean oil, Span 60

(C) 2019 The Authors. Published by Innovare Academic Sciences Pvt Ltd. This is an open access article under the CC BY license (http://creativecommons.org/licenses/by/4.0/D DOI: http://dx.doi.org/10.22159/ijap.2019v11i5.33987

\section{INTRODUCTION}

Gels are semi-solid bases in which a liquid phase is entrapped within a three dimensionalpolymeric network forming a supra-molecular architecture which has a wide range of applications in medicine, biomaterials, cosmetics and food industries. In general, depending on the polarity of the liquid phase, gels have been categorized as either hydrogels (polar solvent: water) or organogels (non-polar solvents: organic liquids, vegetable oils etc.) [1]. Hydrogels have extensive applications in tissue engineering, food and pharmaceutical industry [2]. They appeal to patients/consumers owing to their non-oily feel, cooling effect and water-washability. But they have limited ability to accommodate wide range of drugs and to cross the lipophilic stratum corneum [3]. Attempts were made to overcome these drawbacks by developing organogels and emulgels.

Organogels were being investigated since early1990s [4, 5]. Emulgels are oil-in-water or water-in-oil emulsions, gelled by mixing with a crosslinker $[6,7]$. But due to their stickiness and oily residues, emulgels and organogels have been less appreciated by consumers. Moreover, the leaching of the internal oil phase from these gels on long-term storage has forced scientists to look for stable formulations [8]. This problemmay be attributed to the mismatch in the mechanical properties of the internal and the external phases. To overcome this problem, the concept of bigels was introduced in 2008 by Almeida et al. [9]. Bigels are biphasic systems like emulsions and emulgels, where both internal and external phases are semisolid in nature [10]. Microscopic study of bigels using sesame oil/soybean oil based organogel and gelatin based emulgel revealed the aggregation of droplets whereas emulgel showed dispersed droplets within the continuous phase. They are reported to show negligible amount of leaching of internal phases as compared to the emulgel and in vitro drug release studies indicated non-Fickian diffusion from the bigel matrices [11]

Bigels can be defined as a mixture of an aqueous gel (hydrogel) and an organogel in a definite ratio $[9,12]$. They differ from emulsions, creams and emulgels as they do not require surfactant-based stabilizer [13]. Oil-based do not show demixing of the two phases on storage at room-temperature for a period of up to 6-12 mo [14] They are stabilized by entrapment of the mobile phases via a threedimensional gel network resulting in an extra-fine dispersion. Electrical conductivity of bigels has been ascribed to the presence of pockets of water [9]. As a pharmaceutical formulation, bigels possess many advantages over other semi-solid systems owing to the synergistic effect of both gels, ease of preparation, satisfactory stability, viscosity, spreadability, microarchitecture, absence of surfactant related skin toxicity and possible delivery of both lipophilic and hydrophilic drugs [15].

The aim of the present study was to develop and characterize organogel-in-hydrogel type bigels of soybean oil-based organogel with HPMCK4M hydrogel. Four different types of bigel formulations have been prepared by choosing two organogels containing 20 and $22 \% \mathrm{w} / \mathrm{v}$ Span 60 as organogelator. They were characterized for in vitro drug release profile for topical application.

\section{MATERIALS AND METHODS}

\section{Materials}

Food grade soybean oil (Emami Ltd., Kolkata) was purchased from local market. Paracetamol IP (PCM) was received as a gift sample from enlisted vendor. Span 60 (sorbitan monostearate) was purchased from Loba Chemie Pvt. Ltd., Mumbai. HPMCK4M was of AR grade and obtained from Colorcon Asia Pvt. Ltd. as gift sample. All other reagents were of analytical grade.

Methods

\section{Method of preparation of gel}

The organogel was prepared by adding required quantity of Span 60 in soybean oil maintained at $60{ }^{\circ} \mathrm{C}$ with continuous stirring (500 rpm). The hot dispersion turned into organogel when left undisturbed and cooled down to $25^{\circ} \mathrm{C}$ The drug-loaded organogel 
was prepared by addition of paracetamol (PCM) followed by addition of Span 60 .

The hydrogel was prepared by stirring a definite quantity of HPMCK4M in warm distilled water $\left(65^{\circ} \mathrm{C}\right)$ at $500 \mathrm{rpm}$ to obtain a viscous dispersion $(3-5 \% \mathrm{w} / \mathrm{v})$. The organogel in sol state was added to HPMC aqueous dispersion maintained at $60-70^{\circ} \mathrm{C}$ under stirring at $500 \mathrm{rpm}$ [16]. The stirring was continued until a homogenous mixture was obtained. The mixture formed bigel when cooled to $25^{\circ} \mathrm{C}$. Propylparaben $(0.02 \% \mathrm{w} / \mathrm{v})$ was added to prevent bacterial contamination of the hydrogel. Compositions of the various formulations are provided in table 1 .

Table 1: Composition of Span 60 based organogels and bigels of soybean oil

\begin{tabular}{|c|c|c|c|c|c|}
\hline \multirow[t]{3}{*}{ Formulation } & \multicolumn{3}{|l|}{ Organogel (OG) } & \multicolumn{2}{|c|}{ Hydrogel(HG) } \\
\hline & \multicolumn{3}{|l|}{$\% w / v$} & \multicolumn{2}{|c|}{$\% w / v$} \\
\hline & Paracetamol & Span 60 & Oil & HPMCK4M & Water \\
\hline OG1 & 2 & 20 & 78 & - & - \\
\hline OG2 & 2 & 22 & 76 & - & - \\
\hline HG1 & - & - & - & 3 & 97 \\
\hline $\mathrm{HG} 2$ & - & - & - & 4 & 96 \\
\hline \multicolumn{6}{|l|}{ (OG: $\mathrm{HG}=1: 1)$} \\
\hline BG1 & 2 & 20 & 78 & 3 & 97 \\
\hline $\mathrm{BG} 2$ & 2 & 20 & 78 & 4 & 96 \\
\hline BG3 & 2 & 22 & 76 & 3 & 97 \\
\hline BG4 & 2 & 22 & 76 & 4 & 96 \\
\hline
\end{tabular}

\section{Characterization of the formulations}

\section{FTIR spectroscopy}

FTIR analysis of blank organogel and hydrogel, as well as drug loaded formulations along with its individual components, was carried out using Fourier Transformed Infrared Spectrometer (ALPHA-II, Bruker, Bellerica, MA, USA) operated in Attenuated Total Reflectance (ATR) mode. Samples were scanned in the range of 4000 to $500 \mathrm{~cm}^{-1}$ [16].

\section{Physical evaluation}

Formulations were observed visually for their colour, appearance, texture and opacity [17].

\section{Determination of $\mathbf{p H}$}

The $\mathrm{pH}$ of the formulations was determined using digital $\mathrm{pH}$ meter (Fisher Scientific-Accumet AE 150) [17].

\section{Extrudability}

A definite weight of gel was filled into an ointment tube and crimped. The extrudability $(\mathrm{cm} / \mathrm{s})$ of gel was determined by measuring the length of the gel ribbon extruded from the ointment tube by applying uniform pressure over a period of $10 \mathrm{~s}$ [18]. The following equation was used to determine extrudability.

Extrudability $=$ Distance travelled by the gel $(\mathrm{cm}) / 10 \mathrm{~s}$ (1)

\section{Spreadability}

Spreadability of the formulations was determined by placing $0.1 \mathrm{~g}$ gel between two glass slides of equal dimensions $(75 \mathrm{~mm} \times 25 \mathrm{~mm} \times 1$ $\mathrm{mm}$ ). Thereafter, known weights of $10 \mathrm{~g}, 20 \mathrm{~g}, 50 \mathrm{~g}$ or $100 \mathrm{~g}$ were loaded on the upper slide for $60 \mathrm{~s}$. The initial and final spreading diameters were marked before and after placing the individual weight [19]. Finally, the \% spreadability may be calculated by using the following equation.

$$
\% \text { Spreadability }=\left[\left(D_{i}-D_{f}\right) / D_{i}\right] \times 100
$$

Where, $\mathrm{D}_{\mathrm{i}}=$ initial spreading diameter, $\mathrm{D}_{\mathrm{f}}=$ final spreading diameter

\section{Rheological study}

The viscosity of the blank gels (organogel and bigel)were measured in Brookfield Digital Viscometer (Model LVDVI+, Brookfield Engineering Laboratories Inc, USA) with spindle no. 7 at $25^{\circ} \mathrm{C}[20]$. The apparent viscosity of organogel and bigel formulations of different compositions was measured as a function of shear rate varying from 1 to $5 \mathrm{rpm}$. Ostwald-de wale power-law model has been employed to analyze the flow behavior of organogel as well as bigel systems given as follows:

$$
\mathrm{T}=\mathrm{k}^{*} \gamma^{\mathrm{n}}
$$

Where the relationship between stress $(\mathbb{C})$ and shear rate $(\gamma)$ give the values for flow consistency index $(\mathrm{k})$ and flow behaviour index (n). The rheological behavior may be stated as non-Newtonian pseudoplastic/shear-thinning if the values of $n$ is $<1[21,22]$.

\section{Thermal analysis}

The gel-sol transition temperature ( $\mathrm{T}_{\mathrm{g}}$ ) of the organogels was determined by falling ball method [23]. Briefly, a metal ball of weight $250 \mathrm{mg}$ was placed gently on the surface of the organogel taken in a beaker. A thermometer was inserted in the gel and the gel was heated from 25 to $70^{\circ} \mathrm{C}$ at a rate of $1{ }^{\circ} \mathrm{C} / \mathrm{min}$. The temperature at which the ball started to move from the surface through the gel was recorded as the gel-sol transition temperature $\left(\mathrm{T}_{\mathrm{g}}\right)$. This method could not be used for bigels due to phase separation occurring simultaneously with phase transition at temperature higher than $50^{\circ} \mathrm{C}$. Bigels are reported to lose their stability and structure at higher temperatures [24].

\section{Drug content determination}

Definite amount of drug-loaded gels was added to phosphate buffer $\mathrm{pH}$ 5.8) which was kept undisturbed for $48 \mathrm{~h}$ for complete leaching of drug [25]. The dispersion was filtered through Whatman filter paper No. 1. An aliquot of filtrate was suitably diluted and absorbance measured spectrophotometrically at $249 \mathrm{~nm}$ (Shimadzu UV-VIS 1800 spectrophotometer) [26]. The drug content of formulations was determined from the calibration curve of the drug in the said buffer.

\section{In vitro drug release study}

In vitro drug release from organogels and bigels was performed through dialysis membrane (HIMEDIA ${ }^{\circledR}$ LA 330-5MT) in modified Franz diffusion cell [27]. Accurately weighed drug-loaded sample containing drug equivalent to $4 \mathrm{mg}$ was placed in the donor compartment and the receptor chamber containing phosphate buffer ( $\mathrm{pH}$ 5.8) was maintained at $32 \pm 0.5^{\circ} \mathrm{C}$. An aliquot of $1 \mathrm{ml}$ was withdrawn every hour, replenished with fresh buffer and study was continued for $7 \mathrm{~h}$. The aliquot was analyzed spectrophotometrically at $249 \mathrm{~nm}$ (Shimadzu UV-VIS 1800 spectrophotometer) [26].

Drug release data were subjected to mathematical modeling by using zero-order, first order, Higuchi and Korsmeyer-Peppas models [28].

\section{Hemocompatibility study}

Accurately weighed amount of blank organogel and bigel was placed in dialysis tube filled with $50 \mathrm{ml}$ normal saline $(0.9 \% \mathrm{w} / \mathrm{v} \mathrm{NaCl}$ solution) and continuously stirred in a magnetic stirrer for $1 \mathrm{~h}$ at $37^{\circ} \mathrm{C}$ in order to enable leaching of the gel components. Leachant $(0.5 \mathrm{ml})$ was withdrawn, diluted to $10 \mathrm{ml}$ with normal saline and $0.5 \mathrm{ml}$ diluted goat blood ( $4 \mathrm{ml}$ of goat blood diluted with $5 \mathrm{ml}$ of normal saline) and incubated at $37^{\circ} \mathrm{C}$ for $1 \mathrm{~h}$. It was then centrifuged at $3000 \mathrm{rpm}$ for 10 min. The supernatant was measured spectrophotometrically at $542 \mathrm{~nm}$. Positive control was prepared by taking $0.1(\mathrm{~N}) \mathrm{HCl}$ solution in lieu of 
leachant. In negative control, normal saline was used instead of leachant. Normal saline was taken as the corresponding blank and percent haemolysis may be calculated as follows [29]:

$\%$ Haemolysis $=\left(O D_{\text {test }}-O D_{\text {negative }}\right) /\left(O D_{\text {positive }}-O D_{\text {negative }}\right) \times 100 \ldots(4)$
Where, $\mathrm{OD}_{\text {test }}=$ Absorbance of the test sample

$\mathrm{OD}$ positive $=$ Absorbance of the positive control

$\mathrm{OD}_{\text {negative }}=$ Absorbance of the negative control
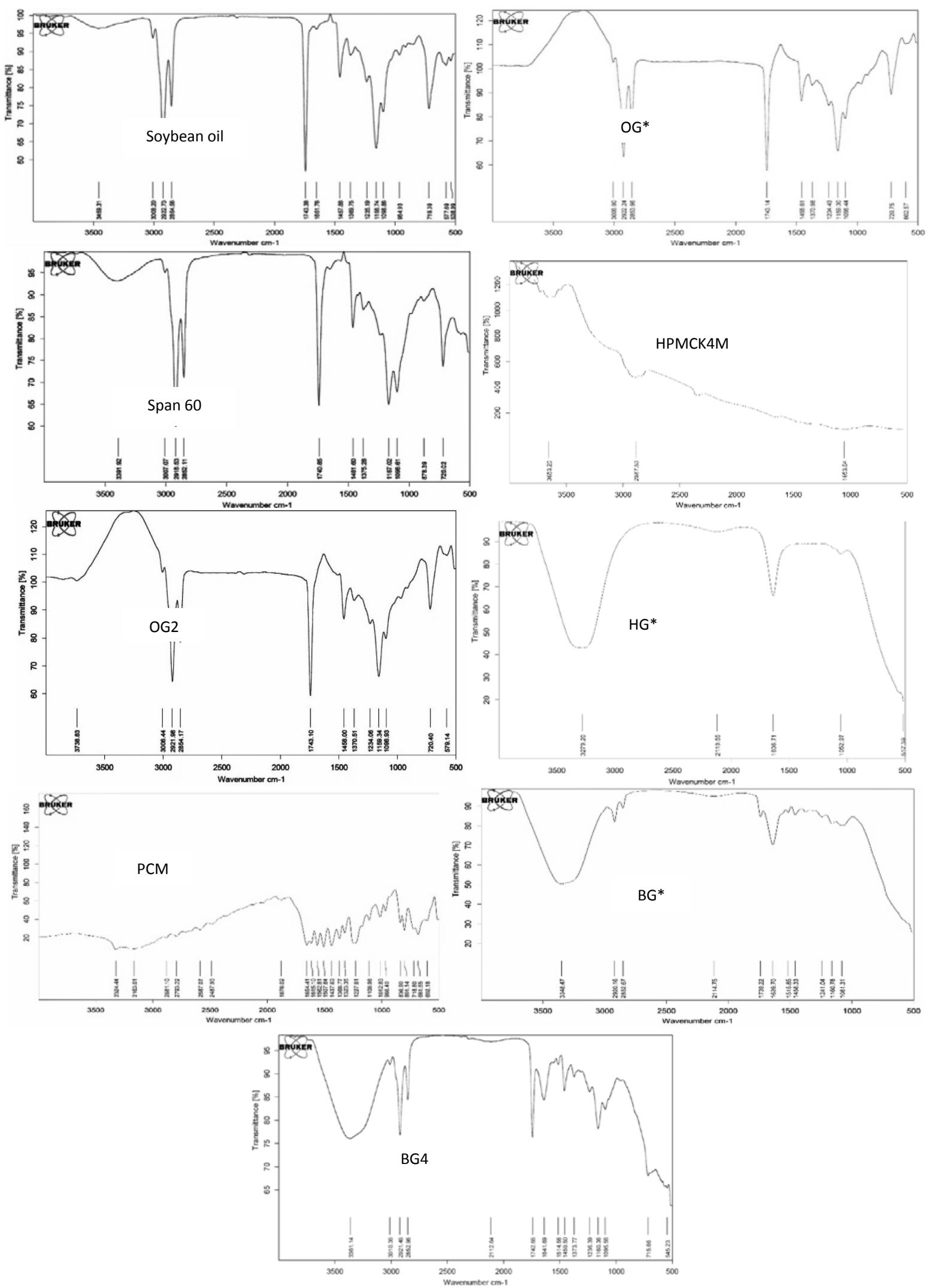

Fig. 1: FTIR analysis of gel components, blank gels (OG* and HG*) and drug-loaded gel (OG2 and BG4) 


\section{Statistical analysis}

All the experiments were performed in triplicate. All data were expressed as mean \pm standard deviation (SD). ANOVA was used to calculate significant differences between the experimental data. The p-value less or equal to 0.05 was considered to be statistically significant [30]

\section{RESULTS}

\section{Formation of gel}

Non-flowing bigel was formed within $10 \mathrm{~min}$ whereas organogel formation occurred in less than $5 \mathrm{~min}$.

\section{FTIR study}

The FTIR spectra of blank organogel $\left(\mathrm{OG}^{*}\right)$ and drug-loaded formulations (OG2 andBG4) are shown in fig. 1. Major peaks of individual components could be detected in the corresponding organogel and bigel. No new peaks could be seen.

\section{Physical evaluation and pH determination}

Organogels appeared to be creamy white in colour with good consistency. Bigels were found to be milky white with smooth, nongreasy and creamy texture. $\mathrm{pH}$ of the formulations was found to be in the range 5.35 to 6.1 at $25^{\circ} \mathrm{C}$ which was close to skin $\mathrm{pH}$ (4.5-6.5) (table 2).

\section{Extrudability and spreadability}

Extrudability and spreadability data of both organogels and bigels are presented in table 3 .

\section{Rheological study}

Viscosities of the gel formulations are graphically represented (fig. 2 ). The flow index ' $n$ ' was found to be less than 1 in all cases.

Table 2: Organoleptic properties and $\mathrm{pH}$ values of Span 60 based organogels and bigels of soybean oil. *Data presented as mean \pm standard error of mean from $n=3$. $p<0.05$ indicating statistically significant differences

\begin{tabular}{|c|c|c|c|c|c|}
\hline Formulation & Colour & Odor & Appearance & Opacity & pH at $25^{\circ} \mathrm{C}^{*}$ \\
\hline OG1 & Creamy white & Odorless & Greasy & Opaque & $5.51 \pm 0.16$ \\
\hline OG2 & Creamy white & Odorless & Greasy & Opaque & $5.26 \pm 0.35$ \\
\hline BG1 & Milky white & Odorless & Smooth non-oily & Opaque & $5.81 \pm 0.47$ \\
\hline BG2 & Milky white & Odorless & Smooth non-oily & Opaque & $5.35 \pm 0.14$ \\
\hline BG3 & Milky white & Odorless & Smooth non-oily & Opaque & $5.98 \pm 0.38$ \\
\hline BG4 & Milky white & Odorless & Smooth non-oily & Opaque & $6.10 \pm 0.32$ \\
\hline
\end{tabular}

Table 3: Extrudability, spreadability and hemocompatibility data of span 60 based organogels and bigels of soybean oil. *Data presented as mean \pm standard error of mean from $n=3$. $p<0.05$ indicating statistically significant differences

\begin{tabular}{|c|c|c|c|c|c|c|}
\hline \multirow[t]{2}{*}{ Formulation } & \multirow[t]{2}{*}{ Extrudability* $(\mathrm{cm} / \mathrm{s})$} & \multicolumn{4}{|c|}{ \% Spreadability on applying } & \multirow[t]{2}{*}{ \% Hemocompatibility } \\
\hline & & $10 \mathrm{~g}$ & $20 \mathrm{~g}$ & $50 \mathrm{~g}$ & $100 \mathrm{~g}$ & \\
\hline OG1 & $0.78 \pm 0.35$ & 37.49 & 45.26 & 74.85 & 92.76 & 3.84 \\
\hline OG2 & $0.76 \pm 0.16$ & 33.33 & 46.15 & 57.89 & 97.43 & 3.52 \\
\hline BG1 & $0.75 \pm 0.24$ & 28.34 & 39.17 & 50.84 & 95.45 & 2.45 \\
\hline BG2 & $0.72 \pm 0.13$ & 24.18 & 32.61 & 45.85 & 92.49 & 3.17 \\
\hline BG3 & $0.78 \pm 0.38$ & 29.59 & 35.48 & 56.17 & 94.25 & 1.35 \\
\hline BG4 & $0.76 \pm 0.34$ & 22.14 & 34.31 & 52.17 & 92.52 & 2.49 \\
\hline
\end{tabular}

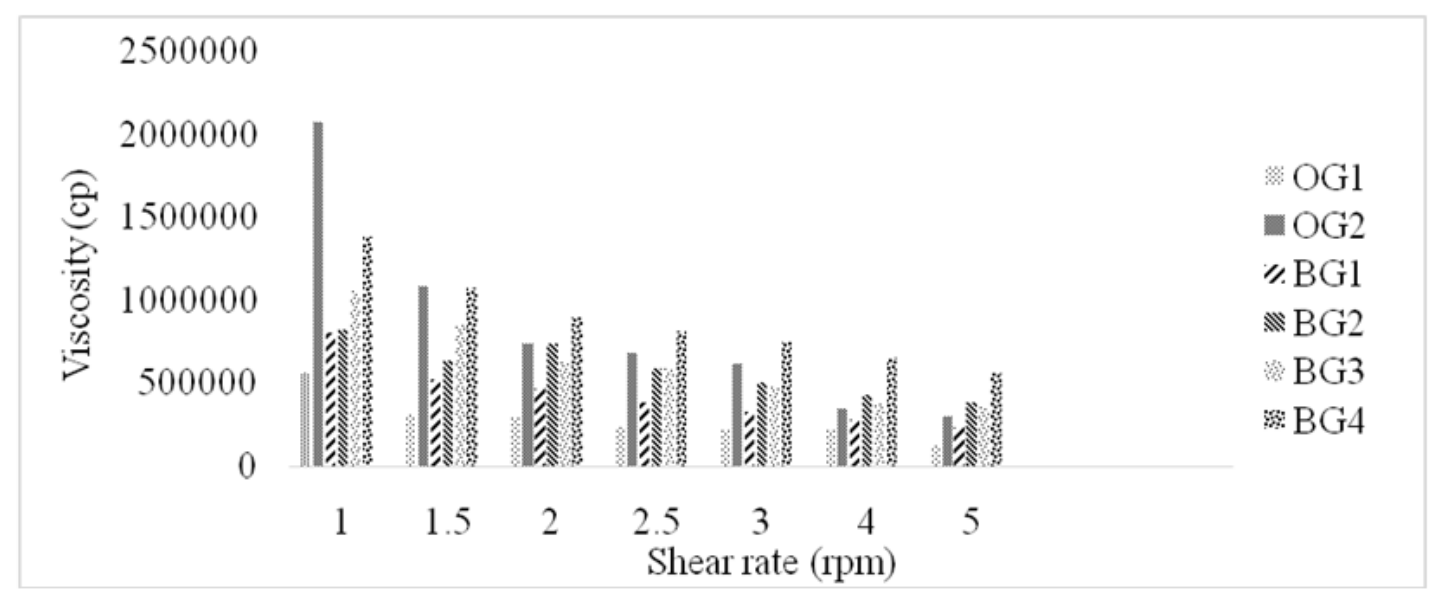

Fig. 2: Shear rate vs viscosity graph of Span 60 based organogels and bigels of soybean oil

\section{Thermal analysis}

Gel-sol transition temperatures of OG1 and OG2 were found to be 59 and $63^{\circ} \mathrm{C}$ respectively.

\section{Drug content determination}

Drug content of organogels (OG1 and OG2) was found to be in between $90-93 \%$ whereas it was found to be in the range $85-92 \%$.

\section{In vitro drug release with kinetic modelling}

Only $\sim 27 \%$ drug release was observed from organogels, OG1 and OG 2 containing 20 and $22 \% \mathrm{w} / \mathrm{v}$ Span 60 respectively while it varied between 40 to $54 \%$ in HPMCK4M based bigels (fig. 3). However, it was noted that an increase in the concentration of HPMC in hydrogel lowered release of PCM from the bigels which was still better than that from organogels. 
Table 4: Modelling of drug release kinetics from Span 60 based organogels and bigels of soybean oil

\begin{tabular}{llllll}
\hline Formulation & \multicolumn{2}{l}{ Zero order model } & \multicolumn{2}{l}{ Higuchi model } & \multicolumn{2}{l}{ Korsmeyer-Peppas model } \\
\cline { 2 - 5 } & $\mathbf{R}^{\mathbf{2}}$ & $\mathbf{R}^{\mathbf{2}}$ & $\mathbf{R}^{\mathbf{2}}$ & $\mathbf{n}$ & Type of diffusion \\
\hline OG1 & 0.997 & - & - & 0.57 & non-Fickian diffusion \\
OG2 & - & - & 0.992 & 0.70 & non-Fickian diffusion \\
BG1 & - & 0.989 & - & 0.23 \\
BG2 & - & 0.978 & - & 0.50 & NP* \\
BG3 & - & 0.947 & - & 0.37 & non-Fickian diffusion \\
BG4 & - & 0.987 & - & 0.57 & NP* \\
\end{tabular}

NP*: not possible to comment

Kinetic modeling of drug release from organogel indicated zeroorder kinetics and Korsmeyer-Peppas model respectively with nonFickian diffusion $(0.45<\mathrm{n}<0.89)$. However, it has been found to follow Higuchi model in the bigels (table 4). In case of bigels with lower \%w/v of HPMC, no decision could be taken regarding the type of diffusion as ' $n$ ' value was found to be out of conventional range.

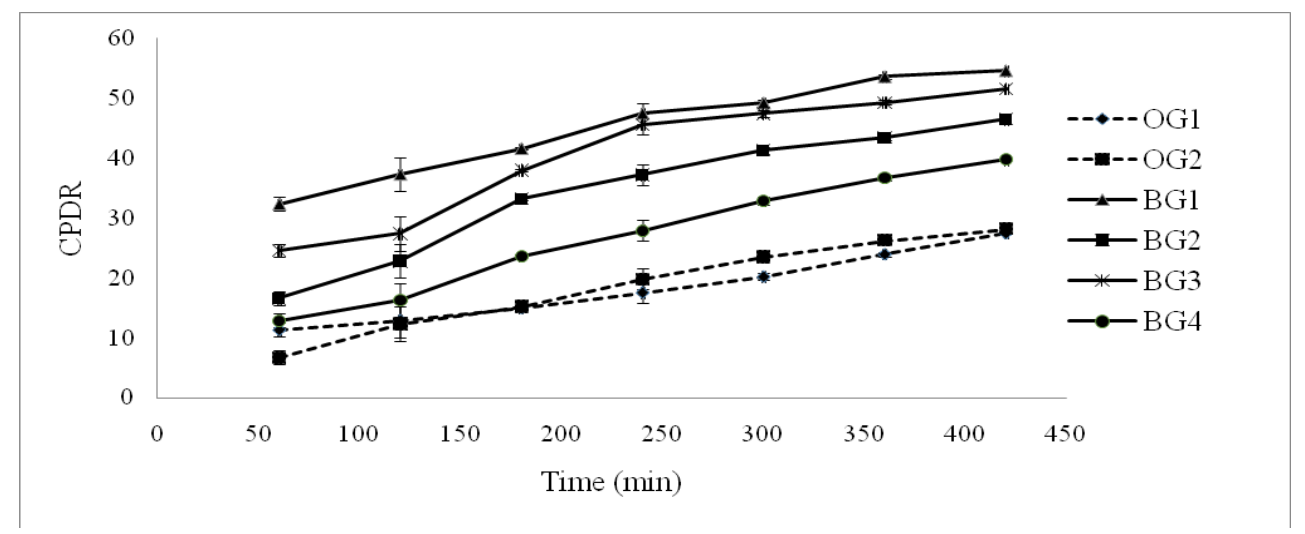

Fig. 3: Drug release from Span 60 based organogels and bigels of soybean oil. Data presented as mean \pm standard error of mean from $n=3$. $\mathbf{p}<\mathbf{0 . 0 5}$ indicating statistically significant differences

\section{Hemocompatibility study}

The \% hemolysis of all the formulations was found to be less than $5 \%$ in presence of organogel and bigel leachant and thus hemocompatible (table 3 ).

\section{DISCUSSION}

Visual appearance and feel of topical preparation is an important characteristic as it affects the choice and compliance of the patient. The smooth and creamy texture of bigels may be attributed to the uniform mixing of otherwise two immiscible phases owing to the presence of Span 60, a surface-active agent [31].

FTIR spectra indicate that the principal peaks present in the raw materials (Soybean oil, Span 60, HPMCK4M and paracetamol) were preserved in the drug-loaded bigels [16].

The viscosities of both organogels and bigels showed concentrationdependent behavior, i.e. increase in viscosity with increase in oraganogelator or aqueous gelling agent (HPMC) concentration. The bigels (BG1 and BG2) formulated with organogel containing 20\% w/v Span (OG1) were found to possess higher viscosity than OG1. However, an anomalous behavior was observed with bigels (BG3 and BG4) of organogel developed with $22 \%$ w/v Span (OG 2) where viscosity is lower than the corresponding organogel. In case of BG3 and BG4 with higher \% of organogelator which is primarily a surfactant, probably an emulgel of lower viscosity would have been formed on addition of 3 and $4 \% \mathrm{w} / \mathrm{v}$ HPMC gels. Lower \% of Span in OG1 did not promote emulgel formulation in the bigels (BG1 and BG 2 ) and hence, their viscosities are higher than the corresponding organogel. Both organogel and bigel formulations demonstrated non-Newtonian pseudoplastic flow and shear thinning behavior similar to that observed with sunflower oil and protein based novel bigels as matrices for drug delivery applications as reported by Behera et al. [21].
As the temperature of the organogels was increased there was a corresponding increase in surface free energy with subsequent increase in mobility of the gelator molecules constituting the 3D-self assembled structure of the formulations, leading to sol formation. Thermal stability of the organogels was enhanced by increasing the concentration of organogelator. [33].

Bigel formation was found to enhance drug release. Drug release via non-Fickian diffusion phenomenon followed either zero-order kinetics from organogel with lower \% $(20 \% \mathrm{w} / \mathrm{v})$ Span or Korsmeyer-Peppas model from organogel with $22 \% \mathrm{w} / \mathrm{v}$ Span. However, bigels are assumed to form matrix as drug release followed Higuchi kinetics. Similar behavior was reported by Rehman et al. (2014) in their studies on polymer-fish oil bigel system as transdermal drug delivery vehicle [32]. It is to be noted that nonFickian diffusion occurred from the organogels and bigels with $4 \% \mathrm{w} / \mathrm{v}$ HPMCK4M irrespective of concentration of organogelator. Improved drug release from bigels can be explained by the swellinginduced gradual break-up of the gel matrix into smaller fragments as the gel skeleton is compromised by the influx of dissolution medium via the channels offered by the tubular structure of gelator molecules. In case of BG 2 and BG 4, swollen but highly dense and compacted structure imparted by higher concentration of HPMC hydrogel presumably failed to cause maximum stressing and expansion of organogel core and thus retarded drug release. Moreover, higher concentration of HPMCK 4M in BG 3 and BG 4 hindered drug release from the gel-matrix due to formation of highviscosity drug diffusion barrier.

\section{CONCLUSION}

From the above studies it can thus be concluded that pseudoplastic bigels with HPMC formed matrix where drug release improved significantly in comparison to Span 60 based organogels of soybean oil. 


\section{AUTHORS CONTRIBUTIONS}

All the author have contributed equally

\section{CONFLICT OF INTERESTS}

Declared none

\section{REFERENCES}

1. Mujawar NK, Ghatage SL, Yeligar VC. Organogel: factors and its importance. Int J Pharm Chem-Bio Sci 2014;4:758-73.

2. Peppas NA, Bures P, Leobandung W, Ichikawa H. Hydrogels in pharmaceutical formulations. Eur J Pharm Biopharm 2000;50:27-46.

3. Mura P, Faucci MT, Bramanti G, Corti P. Evaluation of transcutol as a clonazepam transdermal permeation enhancerfrom hydrophilic gel formulations. Eur J Pharm Sci 2000;9:365-72.

4. Willimann H, Walde P, Lusi PL, Gazzaniga A, Stroppo F. Lecithin organogel as matrix for transdermal transport of drugs. J Pharm Sci 1992;92:871-4.

5. Sagiri SS, Behera B, Rafanan RR, Bhattacharya C, Pal K, Banerjee I, et al. Organogels as matrices for controlled drug delivery: a review on the current state. Soft Materials 2014;12:47-72.

6. Mohamed MI. Optimization of chlorphenesin emulgel formulation. AAPS J 2004;6:1-7.

7. Dickinson E. Emulsion gels: the structuring of soft solids with protein-stabilized oil droplets. Food Hydrocolloids 2012;28:224-41.

8. Chen H, Chang X, Dub D, Li J, Xua H, Yang X. Microemulsionbased hydrogel formulation of ibuprofen for topical delivery. Int J Pharm 2006;315:52-8.

9. Almeida IF, Fernandes AR, Fernandes L, Ferreira MRP, Costa PC, Bahia MF. Moisturizing effect of oleogel/hydrogel mixtures. Pharm Dev Tech 2008;13:487-94.

10. Satapathy S, Singh VK, Sagiri SS, Agarwal T, Banerjee I, Bhattacharya MK, et al. Development and characterization of gelatin-based hydrogels, emulsion hydrogels, and bigels: a comparative study. J Appl Polym Sci 2015;132:1-12.

11. Sagiri SS, Singh VK, Kulanthaivel S, Banerjee I, Basak P, Bhattachrya MK, et al. Stearate organogel-gelatin hydrogel based bigels: physicochemical, thermal, mechanicalcharacterizations and in vitro drug delivery applications. J Mech Bio Mat 2014;43:1-34.

12. Ibrahim MM, Hafez SA, Mahdy MM. Organogels, hydrogels and bigels as transdermal deliverysystems for diltiazem hydrochloride. Asian J Pharm Sci 2013;8:48-57.

13. Rhee GJ, Woo JS, Hwang SJ, Lee YW, Lee CH. Topical oleohydrogel preparation of ketoprofen with enhanced skin permeability. Drug Dev Ind Pharm 1999;25:717-26.

14. Michele LD, Fiocco D, Varrato F, Sastry S, Eiser E, Foffi G. Aggregation dynamics, structure, and mechanical properties of bigels. Soft Matter 2014;10:3633-48.

15. Andonova VY, Peneva PT, Apostolova EG, Dimcheva TD, Peychev ZL, Kassarova MI. Carbopol hydrogel/sorbitan monostearate-almond oilbased organogel biphasic formulations: preparation and characterization of the bigels. Trop J Pharm Res 2017;16:1455-63.
16. Singh VK, Anis A, Al-Zahrani SM, Pradhan DK, Pal K. FTIR, electrochemical impedance and iontophoretic delivery analysis of guar gum and sesame oil-based bigels. Int J Electrochem Sci 2014;9:5640-50.

17. Banks SR, Pygall SR, Bajwa GS, Doughty SW, Timmins P, Melia $\mathrm{CD}$. The influence of substituted phenols on the sol: gel transition of hydroxypropyl methylcellulose (HPMC) aqueous solutions. Carbohydrate Polymers 2014;101:1198-104.

18. Mohanan S, Rasheed N, Raj KSB. Formulation and evaluation of antimicrobial gels for the treatment of paronychia. Int J Appl Pharm 2018;10:161-7.

19. Aejaz A, Azmail K, Sanaullah S, Mohsin AA. Formulation and in vitro evauluation of aceclofenac solid dispersion incorporated gels. Int J Appl Pharm 2010;2:7-12.

20. Patel AR, Schatteman D, Vos WHD, Lesaffer A, Dewettinc K. Preparation and rheological characterization of shellac oleogels and oleogel-based emulsions. J Coll Int Sci 2013;411:114-21.

21. Behera B, Sagiri SS, Pal K, Pramanik K, Rana UA, Shakir I, et al. Sunflower oil and protein-based novel bigels as matrices for drug delivery applications-characterization and in vitro antimicrobial efficiency. Pol-Plas Tech Eng 2015;54:837-50.

22. Singh VK, Banerjee I, Agarwal T, Pramanik K, Bhattacharya MK, Pal K. Guar gum and sesame oil based novel bigels for controlled drug delivery. Coll Sur B: Bio Int 2014;123:582-92.

23. Takahashi A, Sakai M, Kato T. Melting temperature of thermally reversible gel. VI. Effect of branching on the sol-gel transition of polyethylene gels. Poly J 1980;12:335-41.

24. Rehman K, Zulfakar MH. Recent advances in gel technologies for topical and transdermal drug delivery. Drug Dev Ind Pharm 2013;40:433-40.

25. Balata GF, Shamard HA El-M. Formulation of chlorpheniramine maleate in Span 60/Tween 20 based organogels for transdermal delivery. J Innov Pharm Bio Sci 2017;4:49-57.

26. Indian Pharmacopoeia: Government of India, Ministry of Health and Family Welfare Department; $7^{\text {th }}$ edition; 2014;1:559-621.

27. Venkateswara RS, Vijaya SP, Padmalatha K. Formulation and evaluation of oxiconazole emulgel for topical drug delivery. Int J Curr Res 2017;9:58876-8.

28. Dash S, Murthy PN, Nath L, Chowdhury P. Kinetic modeling on drug release from controlled drug delivery systems. Acta Pol Pharma Drug Res 2010;67:217-23.

29. Beheraz B, Singh VK, Kulanthaivel S, Bhattacharya MK, Paramanik K, Banerjee I, et al. Physical and mechanical properties of sunflower oil and synthetic polymers based bigels for the delivery of nitroimidazole antibiotic-a therapeutic approach for controlled drug delivery. Eur Pol J 2015;64:253-64.

30. Khiljee T, Akhtar N. Development and in vitro evaluation of a new topical o/w emulgel from fruit extract of pyruscommunis. Int J Pharm Pharm Sci 2019;11:75-9.

31. Charyulu NR, Muaralidharan A, Sandeep DS. Design and evaluation of bigels containing flurbiprofen. Res J Pharm Tech 2018;11:143-52.

32. Rheman K, Md Amin MCI, Zulfakar NF. Development and physical characterization of polymer-fish oil bigel (hydrogel/oleogel) system as a transdermal drug delivery vehicle. J Oleo Sci 2014;63:961-70.

33. Nadeen J, Heenan RK, Murdan S. Amphiphilogels for drug delivery: formulation and characterization. Pharm Res 2004;4:1852-61. 